\title{
Perfil social y económico del Ejido Naica
}

Economic and social profile of the Naica Ejido

\author{
Magaña Magaña José Eduardo ${ }^{1 凶}$, Villarreal Ramírez Víctor Hugo ${ }^{1}$, Kiessling Davison Christian Mauricio ${ }^{1}$, \\ Escalera Ochoa Laura ${ }^{1}$ y Hermosillo Nieto Javier ${ }^{1}$ \\ ${ }^{1}$ Facultad de Ciencias Agrícolas y Forestales-Universidad Autónoma de Chihuahua \\ ${ }^{\bowtie}$ Autor para correspondencia
}

Recibido: $15 / 07 / 2017$

Aceptado: 10/12/2017

\section{RESUMEN}

El presente perfil social y económico se llevó a cabo en el Ejido de Naica, ubicado en Saucillo, Chihuahua. Una vez recabada la información de los cuestionarios paso a ser procesada por el programa estadístico SPSS 23. Se determinaron los datos generales como edad, nivel de estudio, y estado civil de las personas respondientes del cuestionario, las actividades de producción, las actividades empresariales formales, así como las informales; las que se realizan dentro del pueblo por las familias y se venden de persona a persona o para autoconsumo, sin tener necesariamente un local o tienda donde se esté registrada con una marca, la situación familiar (ingresos familiares), el destino del ingreso y la intención de emprendedurismo; proyectos potenciales del ejido. Los resultados permitieron identificar las empresas o proyectos potenciales de carácter familiar, ejidal y personal de la comunidad. Así como también los tipos de apoyos que existen para poder realizar un proyecto de inversión ya sean de Gobierno del estado, Municipio, Fundaciones, SAGARPA, y otros. Se identificó también que las familias que realizan algún proceso de producto alimenticio, en su gran mayoría, es para autoconsumo y/o para venta informal. Las personas que contestaron los cuestionarios en su mayoría son personas de la tercera edad y a los proyectos a los que se les dio más importancia son de carácter ejidal; como la producción del carbón y la goma de mezquite.

Palabras clave: Perfil, Tercera edad, Ingresos, Materiales de construcción y Económico.

\begin{abstract}
ABSTRAC
A social and economic profile was conducted in the Ejido of Naica, located in Saucillo, Chihuahua. Once collected the information from the questionnaires it was processed by the statistical program SPSS 23. General data was determined such as age, level of study, and marital status from the respondents of the questionnaire Besides that, production and formal business activities, as well as the informal which are carried out within the village for families and sold person to person or for self-consumption, without necessarily having a room or tent where it is registered with a brand, family situation (household income). The income and the intention of entrepreneurship; potential projects of the ejido, allowed to identify the companies or potential projects of family, community and individual character of the community. As well as the types of supports that exist to carry out an investment project already be federal Government, Government of the State, municipality, foundations, and others. Also, families that perform some process
\end{abstract}


of food, in its vast majority, identified was for self-consumption or for informal sale. People who answered the questionnaires for the most are the elderly and projects was given to which more importance are cooperative in nature; as the production of carbon and the mesquite rubber.

Keywords: Profile, Third age, Income, Building Materials and Economic Situation.

\section{INTRODUCCIÓN}

Debido al cierre de la mina, el ejido se ha ido a la baja económicamente, ya que la mina era la principal fuente de empleo. Muchos trabajadores fueron despedidos y tuvieron que migrar con sus familias a otros municipios o estados en busca de trabajo. Esto ha ocasionado que negocios locales de Naica disminuyan sus ventas.

Las familias que han decidido quedarse a vivir en Naica, en su mayoría son por personas de la tercera edad, que una gran parte de ellos son pensionados o ejidatarios que no tienen agua para producir cultivos, el ejido de Naica fue conocido dentro del estado por su producción de alfalfa, avena, zacate ray grass y maíz.

El ejido, no tiene información actualizada respecto al desarrollo social y económico de sus miembros. Razón por la cual, el presente estudio tiene como propósito obtener información de las familias pertenecientes al ejido para que sirva de base a otros proyectos de desarrollo.

\section{MATERIALES Y MÉTODOS}

El perfil social y económico se realizó en el ejido Naica, donde nos reunimos con el presidente del comité de vigilancia Eliseo Terrazas y las familias pertenecientes al pueblo. Se aplicaron los cuestionarios en la sala de actos y en los domicilios. Los cuestionarios están conformados con preguntas de carácter social como económico, como son:

Datos generales, Actividad de producción, Actividad empresarial formal, Situación familiar (ingresos familiares), Destino del ingreso y Intención de emprendedurismo.

Generalidades
1. Nombre de la persona entrevistada con derecho ejidal.

2. ¿Cuál es su edad? Años

3. ¿Cuántos años hace que usted vive en esta comunidad?

4. A su juicio, cuantas personas, ha tenido esta comunidad en los siguientes periodos: Hace 11 años (2006) Hace 7 años (2010)___ En el 2016

5. ¿Cuál es su nivel de estudios?

6. ¿Cuántos son de familia?

7. Estado civil?

8. ¿Quién es el jefe de la familia?

9. La casa donde vive es: propiedad privada ejidal

10. ¿De cuál material está construida su casa?

11. ¿Con que servicios cuenta?

12. ACTIVIDAD DE PRODUCCIÓN; Tenencia de la tierra.

13. ¿Qué cultivos siembra usted y su familia?

14. ¿Industrializa o hace algún proceso de productos alimenticios?

15. ¿Cuál es el destino de esa producción casera? Autoconsumo $\%$ Venta__ $\%$

16. En caso de venta, ¿a quienes se los vende?

17. ¿Cuánto representaría en su ingreso familiar?

18. ¿Qué animales tiene en patio o en su terreno?

19. ¿Qué producto(s) realiza usted con sus animales?

20. En caso de venta, ¿a quienes se los vende? 
21. ¿Cuánto representaría en su ingreso familiar?

22. ¿Qué actividad empresarial realiza? En caso de realizarla

23. Los ingresos de su familia mensual ascienden a: $2 \mathrm{mil} \mathrm{a} 5 \mathrm{mil}$

8 mil 8001 a 10mil

10,001 a $14 \mathrm{mil} \_$mayor $\mathrm{de}$
$14 \mathrm{mil}$

24. De los ingresos familiares díganos que porcentaje proviene por mes de: a) Envíos de dinero de familiares de USA, b) Maquila, c) Hogar, d) Agrícola, e) Pecuaria, f) Pensión.

25. ¿Cuánto gasta usted al mes para su hogar?

26. ¿Qué tipo de proyecto le gustaría emprender con la experiencia de su producción familiar?

27. ¿Conoce de algún apoyo para la realización de su actividad

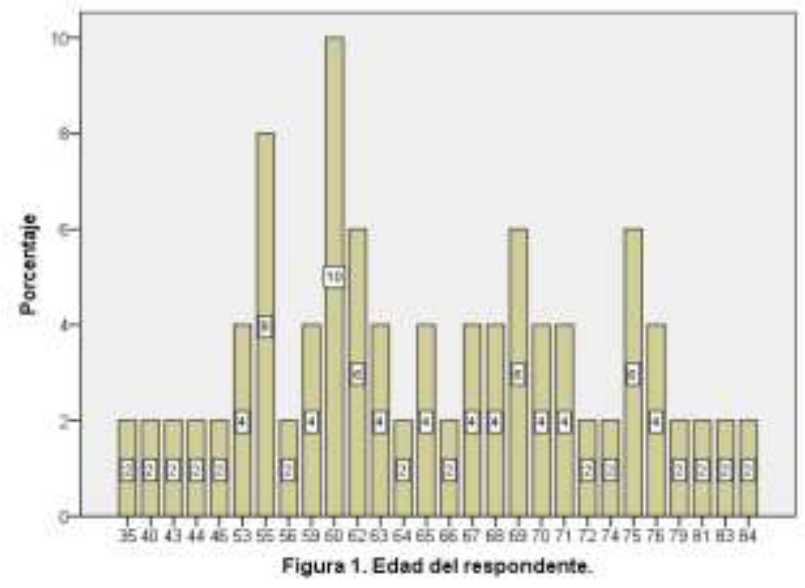

La percepción de las personas cuestionadas acerca del número de habitantes de hace diez años en el pueblo dice que un $40 \%$ contestó que 6000 habitantes, un 14\% dice que habían 4000 habitantes, un $12 \%$ dice que 5000 habitantes y un $10 \%$ dice que contaban con 8000 habitantes (Figura 2).

La percepción acerca de hace cinco años muestra que un $40 \%$ respondió que se contaban con 5000 habitantes, un $34 \%$ dice que 6000 empresarial?

28. Estaría usted y su familia dispuestos a recibir apoyo para la realización de un proyecto que conduzca de la actividad anteriormente mencionada.

Una vez recabada la información de los cuestionarios, se procesó mediante el uso del programa estadístico SPSS.

\section{RESULTADOS}

Después de haber sido recabada la información y procesada en el programa estadístico SPSS, se determinó que las personas que respondieron el cuestionario en su mayoría son personas de la tercera edad, en donde el $16 \%$ son personas de 60 años o más y seguidas con el $8 \%$ de 55 años (Figura 1).

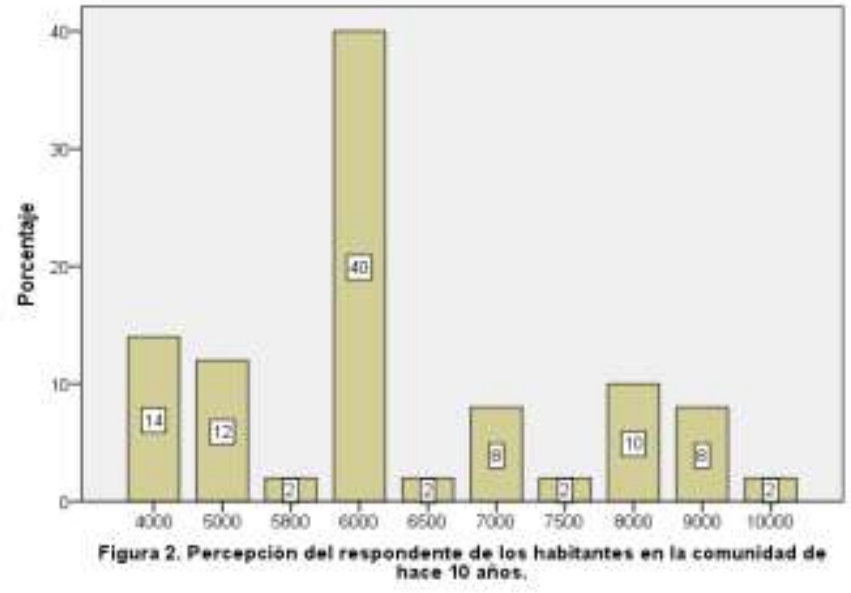

habitantes, mientras que con un $4 \%$ las opiniones están dividas entre 3500, 4000 y 8000 habitantes (Figura 3).

La percepción de hace un año muestra que un $26 \%$ contesto que contaban con 4000 habitantes, un $18 \%$ dice que contaban con 5000 habitantes y un $14 \%$ dice que se contaban con 3000 habitantes (Figura 4). 

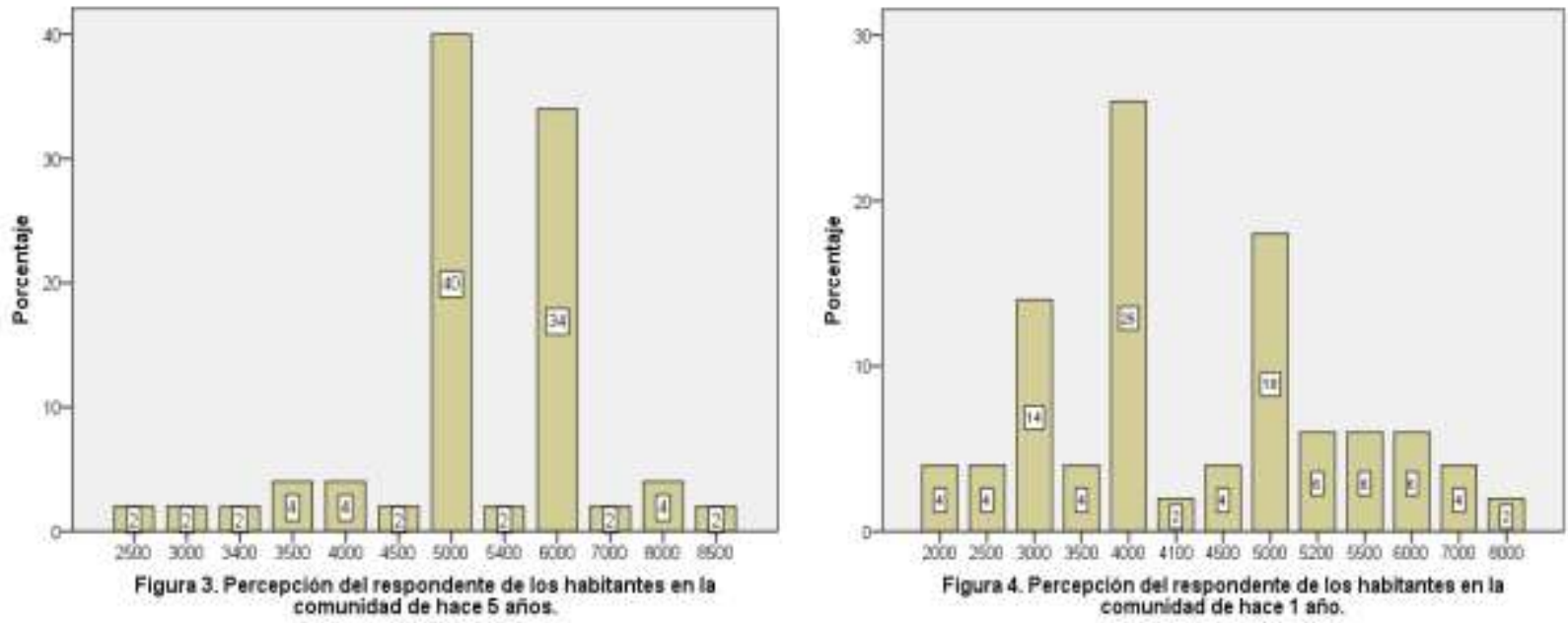

El nivel de estudios de la población de Naica es muy básico ya que el $48 \%$ de ellos solo cuenta con primaria, un $18 \%$ cuenta con el nivel de secundaria, mientras que solo un $6 \%$ cuenta con preparatoria (Figura 5).

Al ser un pueblo casi habitado por puras

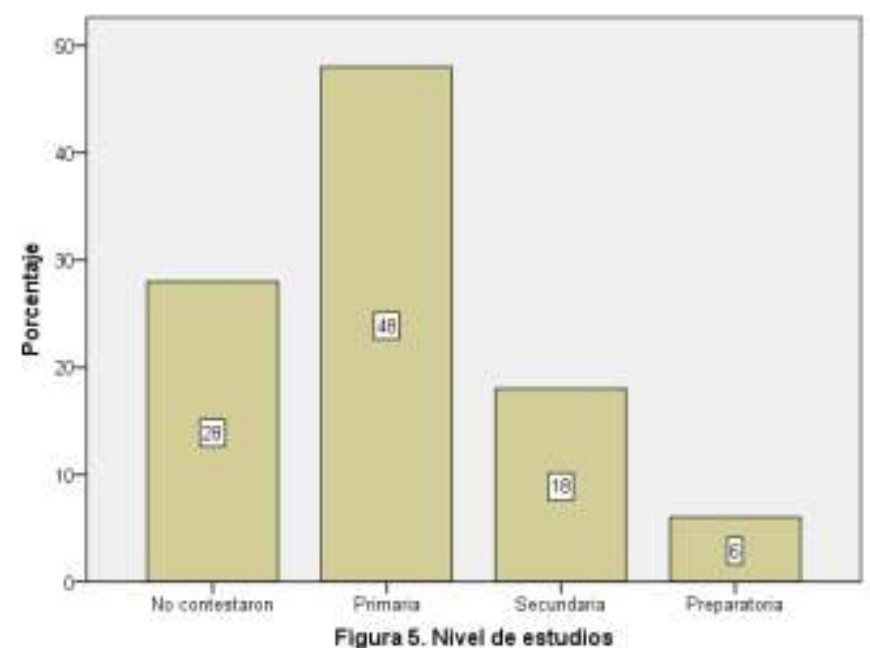

La casa donde viven las familias pertenecientes al pueblo un $66 \%$ es de personas de la tercera edad, el estado civil dice que con un $66 \%$ son casados(as), un $14 \%$ es soltero(a) en su mayoría por personas que nunca se casaron o gente joven que aún no lo hace, un $10 \%$ es viudo(a), un $6 \%$ vive en unión libre y un $4 \%$ es divorciado(a) (Figura 6).

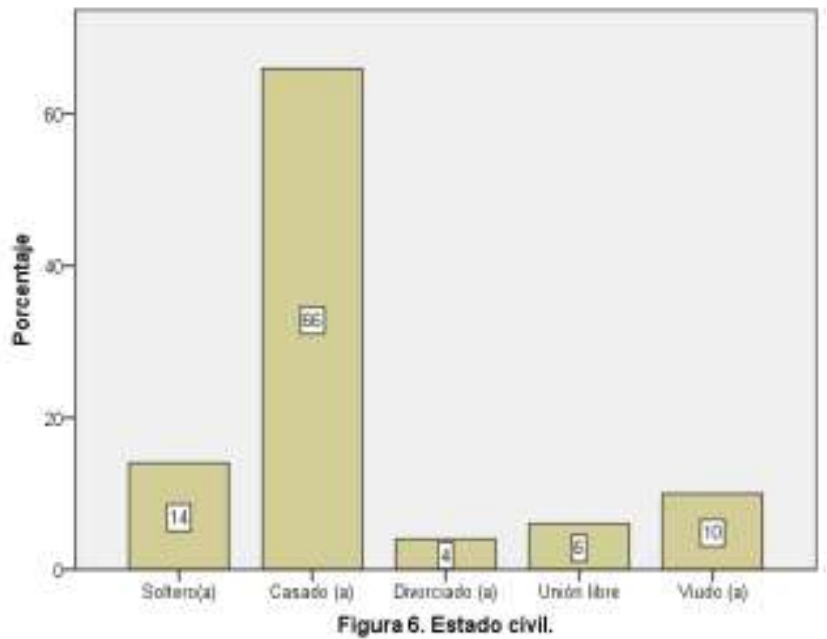

propiedad privada y un $34 \%$ es de propiedad ejidal (Figura 7). 
Tabla 1. El material con el que están construidas las casas es muy importante, ya que son casas que cuentan con muchos años y algunas están hechas con materiales que ya no se producen mucho tales como el adobe.

\begin{tabular}{|l|l|}
\hline MATERIAL & \% CASAS CONSTRUIDAS CON EL MATERIAL. \\
\hline Block & Un 68\% de las casas están construidas con block y un 32\% sin él. \\
\hline Ladrillo & Un $14 \%$ de las casas están construidas con ladrillo y un $86 \%$ sin este material. \\
\hline Adobe & Un 64\% son casas construidas con adobe y un 36\% sin él. \\
\hline Lamina & Casas construidas con lámina son un 76\% mientras que sin ella es un 24\%. \\
\hline Losa & Casas construidas con losa: 30\%, y 70\% están construidas sin ella. \\
\hline $\begin{array}{l}\text { Vigas y } \\
\text { tableta }\end{array}$ & $\begin{array}{l}\text { Casas construidas con vigas y tabletas son un } 40 \% \text { mientras que las casas están } \\
\text { construidas sin ellas son un 60\%. }\end{array}$ \\
\hline Piso de tierra & $\begin{array}{l}\text { Casas construidas con piso de tierra hay un } 8 \%, \text { mientras que un 92\% si cuenta con } \\
\text { piso de otro material. }\end{array}$ \\
\hline $\begin{array}{l}\text { Piso de } \\
\text { cemento }\end{array}$ & $\begin{array}{l}\text { Un } 64 \% \text { de las casas están construidas con piso de cemento, mientras que un 36\% } \\
\text { están construidas sin este material. }\end{array}$ \\
\hline $\begin{array}{l}\text { Azulejo o } \\
\text { vitropiso }\end{array}$ & $\begin{array}{l}\text { Un } 26 \% \text { de las casas cuentan con azulejo y vitropiso, un 74\% no cuenta con este } \\
\text { tipo de material. }\end{array}$ \\
\hline $\begin{array}{l}\text { Otros } \\
\text { Solo un 2\% cuenta con otro tipo de material como cartón por ejemplo y un 98\% no } \\
\text { cuenta con él. }\end{array}$ \\
\hline
\end{tabular}

Tabla 2. Servicios con los que cuentan los hogares y que porcentaje de ellos los tienen.

\begin{tabular}{|l|l|}
\hline SERVICIOS & $\begin{array}{l}\text { PORCENTAJE DE CASAS EN EL EJIDO QUE CUENTAN CON LOS } \\
\text { SERVICIOS }\end{array}$ \\
\hline Luz & El 94\% de las casas cuentan con servicio de luz, un 6\% no cuenta con él. \\
\hline Agua potable & $\begin{array}{l}\text { Casas con servicio de agua potable son un 98\% del pueblo mientras que un 2\% no } \\
\text { cuenta con este tipo de servicio. }\end{array}$ \\
\hline Drenaje & Un 90\% cuenta con drenaje mientras que un 10\% cuenta con fosa séptica. \\
\hline Teléfono & $\begin{array}{l}\text { Casas con servicio de telefonía tales como Telmex, Unefon, etc. Son solo un 28\% un } \\
72 \% \text { no adquiere este tipo de servicio. }\end{array}$ \\
\hline Estufa de gas & $\begin{array}{l}\text { Casas que cuentan con estufa de gas son un 96\%, un 4\% cuenta con estufa de otro } \\
\text { tipo. }\end{array}$ \\
\hline Estufa de leña & Un 30\% de las casas cuentan con estufa de leña, el 70\% no cuenta con este servicio. \\
\hline $\begin{array}{l}\text { Estufa de } \\
\text { petróleo }\end{array}$ & $\begin{array}{l}\text { Casas que cuentan con estufa de petróleo son un 4\% aunque la cifra es baja para la } \\
\text { actualidad es increíble que hayan casas que aún la utilicen, un 96\% no cuenta con este } \\
\text { servicio. }\end{array}$ \\
\hline $\begin{array}{l}\text { Servicio de } \\
\text { televisión }\end{array}$ & $\begin{array}{l}\text { Casas con servicio de televisión como Izzi, Dish, Sky, etc son un 74\% mientras que } \\
\text { un 26\% no adquiere este servicio. }\end{array}$ \\
\hline
\end{tabular}


Tabla 3. Actividades económicas formales e informales que se realizan dentro del ejido.

\begin{tabular}{|l|c|l|c|}
\hline TIPO DE NEGOCIO & FORMALES & TIPO DE NEGOCIO & INFORMALES \\
\hline Agroindustria & & Salsas & $\checkmark$ \\
\hline Tienda de abarrotes & $\checkmark$ & Envasado de chile jalapeño & $\checkmark$ \\
\hline Papelería & $\checkmark$ & Envasado de chiles hueritos & $\checkmark$ \\
\hline Carnicería & $\checkmark$ & Envasado de duraznos & $\checkmark$ \\
\hline Tortillería & & Conserva de calabaza & $\checkmark$ \\
\hline Estética & & Mermelada de membrillo & $\checkmark$ \\
\hline Quesería & & Chile pasado & $\checkmark$ \\
\hline Tortillas de harina & & Chacales & $\checkmark$ \\
\hline Artesanías & & Chile seco & $\checkmark$ \\
\hline Dulces artesanales & & Venta de ropa y cosméticos & $\checkmark$ \\
\hline Panadería & & Carne de cerdo & $\checkmark$ \\
\hline Venta de tamales & & Leche de vaca & $\checkmark$ \\
\hline Otra actividad & $\checkmark$ & Queso & $\checkmark$ \\
\hline & & Asaderos & $\checkmark$ \\
\hline & & Quesos frescos & $\checkmark$ \\
\hline & & Carne de gallina & $\checkmark$ \\
\hline & & Huevo & $\checkmark$ \\
\hline & & Carne de borrego & $\checkmark$ \\
\hline & & Leche de chiva & $\checkmark$ \\
\hline & & & \\
\hline & & & \\
\hline & & & \\
\hline & & & \\
\hline & & & \\
\hline & & & \\
\hline & & & \\
\hline & & & \\
\hline & & & \\
\hline
\end{tabular}

Tabla. 4. Productos que se realizan dentro del pueblo para venta y/o autoconsumo y que porcentaje de familias lo realizan.

\begin{tabular}{|l|r|}
\hline PRODUCTOS & \% DE FAMILIAS \\
\hline Salsas & $10 \%$ \\
\hline Envasado de chile jalapeño & $8 \%$ \\
\hline Envasado de chile huerito & $10 \%$ \\
\hline Envasado de duraznos & $10 \%$ \\
\hline Mermelada de higo & $0 \%$ \\
\hline Conserva de calabaza & $6 \%$ \\
\hline Mermelada de membrillo & $2 \%$ \\
\hline
\end{tabular}

Revista Científica Biológico Agropecuaria Tuxpan 5 (2) 


\begin{tabular}{|l|c|}
\hline Mermelada de mezquite & $0 \%$ \\
\hline Ate de membrillo & $0 \%$ \\
\hline Chile pasado & $2 \%$ \\
\hline Chacales & $8 \%$ \\
\hline Chile seco & $2 \%$ \\
\hline Otros: Quesos frescos, jamoncillos y asaderos. & $4 \%$ \\
\hline
\end{tabular}

Tabla 5. Ingresos familiares mensuales.

\begin{tabular}{|l|lr|}
\hline $\begin{array}{l}\text { INGRESOS FAMILIARES } \\
\text { MENSUALES }\end{array}$ & $\begin{array}{l}\text { \% DE LAS FAMILIAS QUE CUENTAN CON EL } \\
\text { INGRESO }\end{array}$ \\
\hline 2mil a 5mil & & $76 \%$ \\
\hline 5001 a $8 \mathrm{mil}$ & & $16 \%$ \\
\hline 8001 a 10mil & & $4 \%$ \\
\hline 10mil o mas & & $0 \%$ \\
\hline
\end{tabular}

Tabla 6. Gastos mensuales familiares se dividen de la siguiente manera:

\begin{tabular}{|c|c|}
\hline SERVICIOS & GASTO \\
\hline Alimento & $\begin{array}{l}\text { Un } 32 \% \text { de las personas gastan } \$ 4000 \text { pesos, un } 20 \% \text { gasta } \$ 3000 \text { pesos, el otro } \\
48 \% \text { gasta } \$ 2000 \text { o más. }\end{array}$ \\
\hline Luz & $\begin{array}{l}\text { Un } 16 \% \text { gasta } \$ 200 \text { pesos de luz mensuales, seguido de un } 14 \% \text { gasta } \$ 300 \text { pesos } \\
\text { mensuales y el otro } 70 \% \text { gasta cifras más varias unos pagan mucho y más. }\end{array}$ \\
\hline Agua & $\begin{array}{l}\text { El gasto mensual de agua dice que un } 26 \% \text { paga } \$ 100 \text { pesos, un } 24 \% \text { paga } \$ 200 \text { y } \\
\text { al otro } 50 \% \text { le varían los pagos es decir como pagan unos más otros menos. }\end{array}$ \\
\hline Internet & $\begin{array}{l}\text { Un } 88 \% \text { de los respondentes dijo que paga cero pesos de internet ya que no cuenta } \\
\text { con este servicio, el otro } 12 \% \text { paga } \$ 180 \text { pesos mensuales o más. }\end{array}$ \\
\hline Teléfono fijo & $\begin{array}{l}\text { Un } 84 \% \text { de los respondentes dijo que paga cero pesos en teléfono fijo o de casa } \\
\text { porque no cuenta con este servicio, el otro } 16 \% \text { paga } \$ 50 \text { pesos o más mensuales. }\end{array}$ \\
\hline $\begin{array}{l}\text { Teléfono } \\
\text { celular }\end{array}$ & $\begin{array}{l}\text { Un } 44 \% \text { de las personas gasta cero pesos mensuales en teléfono celular porque no } \\
\text { cuenta con uno, el otro } 56 \% \text { gasta mensualmente entre } \$ 50 \text { pesos de recarga hasta } \\
\text { un plan de } \$ 600 \text { pesos. }\end{array}$ \\
\hline Cable & $\begin{array}{l}\text { Un } 42 \% \text { gasta cero pesos mensuales en cable porque no cuenta con el servicio, un } \\
34 \% \text { gasta } \$ 200 \text { pesos y el otro } 24 \% \text { paga entre } \$ 100 \text { y } \$ 420 \text { pesos mensuales. }\end{array}$ \\
\hline Antenas de tv & $\begin{array}{l}\text { Un } 92 \% \text { de las personas no gastan en antenas de televisión, el otro } 8 \% \text { gasta } \$ 200 \\
\text { pesos mensuales o más. }\end{array}$ \\
\hline
\end{tabular}

Revista Científica Biológico Agropecuaria Tuxpan 5 (2) 


\begin{tabular}{|l|l|}
\hline Gasolina & $\begin{array}{l}\text { El gasto mensual en gasolina varía mucho un 38\% paga cero pesos porque no } \\
\text { cuenta con automóvil, y el otro 62\% paga entre \$100 y \$3200 pesos mensuales. }\end{array}$ \\
\hline Gas & $\begin{array}{l}\text { En gas un 22\% gasta cero pesos mensuales porque cuenta con estufa de leña, } \\
\text { petróleo o boiler de leña, el otro 78\% paga entre \$100 y \$4000 pesos mensuales. }\end{array}$ \\
\hline $\begin{array}{l}\text { Pasaje del } \\
\text { camión }\end{array}$ & $\begin{array}{l}\text { Un 82\% gasta cero pesos mensuales en pasaje de camión porque tiene auto propio } \\
\text { o no es necesario en su vida diaria el uso del camión, el otro 18\% paga entre \$100 } \\
\text { y \$1200 pesos mensuales en pasaje de camión. }\end{array}$ \\
\hline $\begin{array}{l}\text { Colegiaturas } \\
\text { pago de } \\
\text { inscripción) }\end{array}$ & $\begin{array}{l}\text { El } 82 \% \text { de los respondentes dijo que gasta cero pesos mensuales en colegiaturas } \\
\text { porque no hay niños y/o jóvenes estudiando en su hogar, el otro 18\% paga entre } \\
\$ 300 \text { y \$1050 pesos mensuales. }\end{array}$ \\
\hline $\begin{array}{l}\text { Vestido } \\
\text { Entretenimie } \\
\text { nto }\end{array}$ & $\begin{array}{l}\text { Un } 72 \% \text { gasta cero pesos mensuales en ropa o vestido, el otro 28\% gasta entre } \\
\text { como una necesidad básica, el otro 14\% gasta entre \$30 y \$1000 pesos. }\end{array}$ \\
\hline
\end{tabular}

\section{DISCUSIÓN}

¿Existen empresas o microempresas dentro del pueblo de Naica? No existe un censo específico que nos indique cuantas empresas hay que cuente con información actualizada, ¿Qué tan factible sería establecer negocios de servicios tales como una compañía de internet, televisión por cable, etc, en el pueblo? No sería factible porque de acuerdo al presente estudio, el $16 \%$ de la población son personas de la tercera edad, seguidos por personas de 55 años (Figura 1), y de acuerdo con la percepción del respondente de hace diez (Figura 2), y hasta hace un año (Figura 4), la población ha ido a la baja, puesto que con el cierre de la mina muchas familias tuvieron que emigrar en busca de mejores oportunidades de trabajo, esto afecto a la economía del pueblo, se cerraron negocios y las personas que viven en el pueblo que no se fueron es porque son personas pensionadas o ejidatarios que no cuentan con agua para producir alguna clase de cultivos y que cuentan con un nivel de estudios muy básico como es la primaria (Figura 5).

\section{LITERATURA CITADA}

Instituto Nacional de Estadística y Geografía (2010). Obtenido de Principales resultados por localidad 2010 ITER

Serca corporativo. (17 de MAYO de 2013). Obtenido de http://corporativoserca.com/blog/que-esun-estudio-socioeconomico-cual-es-elcontenido-y-que-tipos-de-socioeconomicosexisten/

El universal. (13 de OCTUBRE de 2015). Obtenido de

http://www.eluniversal.com.mx/articulo/carte $\mathrm{ra} /$ negocios/2015/10/13/penoles-cierra-lamina-naica-en-chihuahua

Badino et al. "NAICA (CHIHUAHUA, MÉXICO). LOS CRISTALES GIGANTES DE LA MINA DE NAICA SE INUNDAN." Enseñanza de las Ciencias de la Tierra, 2016, Vol. 24, Núm. 1, p. 121-123. Obtenido de http://www.raco.cat/index.php/ECT/article/vi ew/312543/402619

Pae. (2016). Obtenido de www.pae.cc/es/estudiossocioeconomicos/

Serca estudios socioeconómicos. (03 de OCTUBRE de 2014). Obtenido de http://corporativoserca.com/blog/que-es-unestudio-socioeconomico-y-para-que-sirve/ 
Revista ejemplode.com. (A. 2013,05. Ejemplo de Estudio socioeconómico. Revista Ejemplode.com. Obtenido 05, 2013, de http://www.ejemplode.com/59-

finanzas/2940-

ejemplo_de_estudio_socioeconomico.html)

\section{Copyright (c) 2017 José E duardo Magaña Magaña, Victor Hugo Villarreal Ramirez, Christian Mauricio Kiessling Davi son, \\ Laura Escalera Ochoa y Javier Hermosillo Nieto}

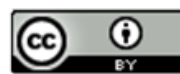

Este tex to está protegido por una licencia licencia Creative Commons 4.0.

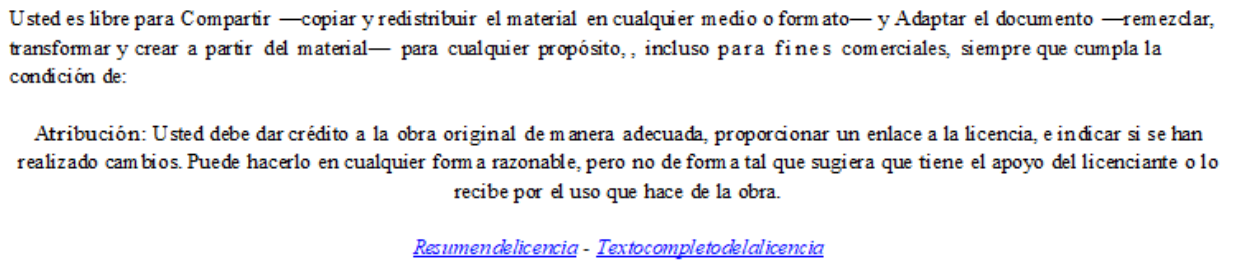

1 documento $\rightarrow$ remezclar, transformar y crear a partir del material- para cualquier propósito, , incluso para fines comerciales, siempre que cumpla la

Restmendelicencia - Textocompletodelalicencia 